\title{
The effects of DNA methylation and histone deacetylase inhibitors upon the human papillomavirus early genes expression in cervical cancer. An in vitro and clinical study
}

\author{
Erik de la Cruz-Hernandez ${ }^{1}$, Adriana Contreras-Paredes ${ }^{1}$, Alejandro Mohar ${ }^{1}$, \\ David Cantú ${ }^{2}$, Marcela Lizano ${ }^{1}$ and Alfonso Duenas-Gonzalez*1
}

Address: ${ }^{1}$ Unidad de Inv. Biomédica en Cancer, IIB, UNAM/INCAN, Mexico and 22Department of Gynecology, INCAN, Mexico

Email: Alfonso Duenas-Gonzalez* - alfonso_duenasg@yahoo.com

* Corresponding author

from $24^{\text {th }}$ Annual Meeting of the National Cancer Institute of Mexico

Mexico City, Mexico. I4-17 February 2007

Published: 5 February 2007

BMC Cancer 2007, 7(SuppI I):A25 doi:10.1 I86/I47I-2407-7-SI-A25

This article is available from: http://www.biomedcentral.com/I47I-2407/7/SI/A25

(C) 2007 de la Cruz-Hernandez et al; licensee BioMed Central Ltd.

\section{Background}

Epigenetic aberrations such as alterations in DNA methylation are a common hallmark of cancer. HPV genome methylation profiling in normal, precursor, and invasive lesion smears has shown that as lesion severity increases, there is progressive hypomethylation on the LCR and E6 gene regions, suggesting that neoplastic transformation can be suppressed by gene hypermethylation, whereas hypomethylation accompanies or causes cervical cancer progression. Because of that, some are argued on caution against the use of epigenetic therapy for HPV-related malignancies as it may act as a tumor promoter by enhancing the expression of HPV oncoproteins. To address this issue, herein we analyzed the effects of DNA methylation and histone deacetylase inhibitors upon the human papillomavirus early genes expression in cervical cancer cell lines and patients.

\section{Methods}

CasKi, HeLa, CaLo, SiHa cell lines were studied. Cell proliferation was assayed by MTT assay. E6/E7 expression by RT-PCR, chromatin immunoprecipitation by ChiP and methylation profile by PCR after McfBC digestion. Clinical samples were taken from clinical studies performed with hydralazine, valproate and both (BMC2005, Mol Cancer 2005, in preparation). P53 expression and p53/E6 interaction were - analyzed by western blot and immunoprecipitation assays.

\section{Results}

Our results demonstrate that hydralazine and valproate induce growth inhibition in cervical cancer cells without causing significant changes in the expression level of E6/ E7 transcript. Likewise, chromatin immunoprecipitation assays demonstrate that hydralazine and valproate induced no major changes in the acetylation status of $\mathrm{H} 4$ at the LCR of the HPV. The lack of significant changes at the expression levels of viral E6/E7 viral oncogenes occurs despite epigenetic drugs induced some degree of hypomethylation at the viral LCR. E6/E7 expression changes were minor and heterogenous in the postreatment biopsies of patients treated with hydralazine, valproate or both. On the contrary, hydralazine and valproate induce a strong increase in p53 at the protein level which resulted from p53 protein acetylation which protects its degradation by E6 leading to transactivation of p53 targets such as the pro-apoptotic gene bax.

\section{Conclusion}

Hydralazine and valproate treatment not only fail to increase HPV E6/E7 viral oncoprotein expresion but leads to accumulation of p53 levels by its acetylation which increases the transactivation function of p53. These data suggest that hydralazine and valproate may increase the efficacy of current cervical cancer treatments. 